\title{
Global wellposedness of cubic Camassa-Holm equations
}

\author{
Qingtian Zhang \\ Department of Mathematics, Penn State University, University Park, Pa. 16802, USA. \\ E-mail: zhang_q@math.psu.edu
}

\begin{abstract}
In this paper, we study the Cauchy problem of cubic Camassa-Holm equation. This equation is derived from a model of shallow water dynamics. We prove the global existence of entropy weak solution to this problem in space $H^{1}$ with its derivative in $B V$. The stability and uniqueness of entropy weak solution are obtained in $W^{1,1}$.
\end{abstract}

\section{Introduction}

We consider the cubic Camassa-Holm equation $(\mathrm{cCH})$ :

$$
m_{t}+\left[\left(u^{2}-u_{x}^{2}\right) m\right]_{x}=0, \quad m=u-u_{x x},
$$

or

$$
u_{t}-u_{x x t}+3 u^{2} u_{x}-u_{x}^{3}-4 u u_{x} u_{x x}+2 u_{x} u_{x x}^{2}+\left(u_{x}^{2}-u^{2}\right) u_{x x x}=0 .
$$

If written as a first-order equation of $u$, it is

$$
u_{t}+u^{2} u_{x}=\frac{1}{3} u_{x}^{3}-\Phi(u)
$$

with

$$
\Phi(u)=\left(1-\partial_{x}^{2}\right)^{-1}\left[\frac{1}{3} u_{x}^{3}\right]+\partial_{x}\left(1-\partial_{x}^{2}\right)^{-1}\left[\frac{2}{3} u^{3}+u u_{x}^{2}\right]
$$

The initial data is

$$
\left.u\right|_{t=0}=u_{0}(x) .
$$

This equation is a complete integrable water wave equation proposed by several different authors $[13,20,21]$. It is a suitable approximation of incompressible Euler system without swirl. For smooth solutions to the equation, we have the following two conserved quantities:

$$
H_{1}(u)=\int_{\mathbb{R}}\left(u^{2}+u_{x}^{2}\right) d x, \quad H_{2}(u)=\int_{\mathbb{R}}\left(u^{4}+2 u^{2} u_{x}^{2}-\frac{1}{3} u_{x}^{4}\right) d x .
$$

In fact, these two invariant quantities are the Hamiltonian functionals of $(\mathrm{cCH})$, with the compatible Hamiltonian operators

$$
\mathcal{J}_{2}=-\partial_{x} m \partial_{x}^{-1} m \partial_{x}, \quad \mathcal{J}_{1}=-\frac{1}{4}\left(\partial_{x}-\partial_{x}^{3}\right) .
$$


With these notations, the equation can be written in the bi-Hamiltonian form

$$
m_{t}=\mathcal{J}_{2} \frac{\delta H_{1}}{\delta m}=\mathcal{J}_{1} \frac{\delta H_{2}}{\delta m} .
$$

In recent decades, the partial differential equations from the fields of integrable systems have been studied adequately. Among them are KdV equation ( $\mathrm{KdV})$, modified $\mathrm{KdV}$ equations $(\mathrm{mKdV})$, generalized $\mathrm{KdV}$ equations $(\mathrm{gKdV})$, Camassa-Holm equations $(\mathrm{CH})$, generalized Camassa-Holm equations ( $\mathrm{gCH}$ ), Degasperis-Procesi equation (DP), and so on [6, 19]. Some of them are completely integrable. They have Lax pair formulation of the equations, which allows to apply inverse scattering techniques [5, 8, 9, 12]. However, not all equations are completely integrable. From analytical point of view, the Hamiltonian quantities usually provide us the functional spaces for the solutions, which is not sufficient to obtain a solution. It usually requires further regularity to guarantee compactness. For example, (gKdV) is a semilinear dispersive equation. In [17], the authors proved global well-posedness by using contract mapping theorem via dispersive estimates.

Camassa-Holm equations are closely related with KdV equations in the sense that CamassaHolm equations are the tri-Hamiltonian duality of KdV equations [20]. However, unlike KdV equations, Camassa-Holm equations do not have that strong dispersive effect. In fact, they are more like quasilinear hyperbolic equations. They have the wave breaking phenomena [7] which is not shared by the KdV-type equations. The problem of global well-posedness of Camassa-Holm equation is equivalent to the problem of how to continue the solution after the singularity appears. There are two methods to deal with it in general: compactness method and method of characteristics. Global existence of weak solutions for $(\mathrm{CH})$ are proved by compensated compactness method in [10] [22], and a result of weak-strong uniqueness is proved in [23]. For (DP), global wellposedness, including existence, uniqueness and stability, is proved in [11]. The other way to obtain global well-posedness for $(\mathrm{CH})$ is by the method of characteristics. In [2] and [3], conservative and dispersive weak solutions are constructed, and in [1], the authors proved the uniqueness of conservative weak solutions.

Different from the Camassa-Holm equation, the cubic Camassa-Holm equation has higher nonlinearity. This difficulty is manifested in the equation of characteristics:

$$
\frac{d x}{d t}=\left(u^{2}-u_{x}^{2}\right)(t, x(t))
$$

From the two conserved Hamiltonians, it is hard to expect the right-hand-side to be continuous with respect to $x$. So this characteristic ODE may not be globally wellposed. The method of characteristics does not apply here.

Here I would like to mention another interesting equation - Novikov equation

$$
u_{t}-u_{x x t}+4 u^{2} u_{x}-3 u u_{x} u_{x x}-u^{2} u_{x x x}=0 .
$$

This equation shares the same conserved quantity with $(\mathrm{cCH})$ but has lower order of nonlinearity. An advantage of this is reflected on the equation of characteristics:

$$
\frac{d x}{d t}=u^{2}(t, x(t))
$$

The right-hand-side is Hölder continuous with respect to $x$. So the method of characteristics works well in this model. For details, see [4].

For the cubic Camassa-Holm equation, local well-posedness of strong solutions has been proved in [14] [15] and [16]. In [15], the authors also revealed the blow-up mechanism of (cCH). They 
proved that $\inf _{x \in \mathbb{R}}\left\{\left(m u_{x}\right)(t, x)\right\}$ will approach $-\infty$ in finite time. It means that the first-order and the second-order derivatives of the solution cannot both be bounded all the time. How to continue the solution after the singularity is an open question, which is our main concern in this paper.

Observe that in $(\mathrm{cCH})$, there is a cubic term of $u_{x}$. This implies that the $(\mathrm{cCH})$ is more like a Hamilton-Jacobi equation, rather than a perturbed hyperbolic conservation law. Based on this observation, we take 1st order derivative to $(\mathrm{cCH})$, and then view the obtained equation as a balance law for $u_{x}$. This suggests us to use the technique in the theory of scalar conservation laws. Fortunately, the source term is under control and we can prove $B V$ estimates of $u_{x}$ from the conservative form of equation of $m$, which leads us to use the Kružhikov's theory [18] to prove global wellposedness.

Denote $X$ to be the functional space

$$
X:=\left\{f \in H^{1}(\mathbb{R}) \cap L^{1}(\mathbb{R}) \mid f_{x} \in B V\right\},
$$

with the norm $\|f\|_{X}=\|f\|_{H^{1}}+\|f\|_{L^{1}}+\left\|f_{x}\right\|_{B V}$.

Our main theorem is:

Theorem 1.1 (Well-posedness of entropy weak solution). Suppose the initial data $u_{0} \in X$. There exists an entropy weak solution to the Cauchy problem (1.3), (1.5), where the definition of the entropy weak solution is in Definition 3.2 in section 3.

The entropy weak solutions have following stability property. Fix any $T>0$, and let $u, v$ : $\mathbb{R}_{+} \times \mathbb{R} \rightarrow \mathbb{R}$ be two entropy weak solutions to (1.3), (1.5) with initial data $u_{0}, v_{0} \in X$, respectively. Then for any $t \in(0, T)$

$$
\|u(t, \cdot)-v(t, \cdot)\|_{W^{1,1}} \leq e^{M_{T} t}\left\|u_{0}-v_{0}\right\|_{W^{1,1}},
$$

where $M_{T}$ is a constant related with $T$ and $H^{1} \cap W^{2,1}$ norms of initial data $u_{0}, v_{0}$. This implies uniqueness.

We have the continuity in time

$$
\left\|u\left(t_{2}, \cdot\right)-u\left(t_{1}, \cdot\right)\right\|_{W^{1,1}} \leq C_{T}\left|t_{2}-t_{1}\right|, \quad \text { for all } t_{1}, t_{2} \in[0, T],
$$

for some constant $C_{T}$. Consequently, the initial data can be interpreted in the following sense:

$$
\lim _{t \rightarrow 0+}\left\|u(t, \cdot)-u_{0}(x)\right\|_{W^{1,1}}=0 .
$$

Remark 1.1. This weak solution includes peakon and anti-peakon solutions with the expression $u(t, x)=a e^{-|x-c t|}, a, c \in \mathbb{R}$.

Remark 1.2. This theorem partially answered the open question proposed in [15]. For an initial data outside $\dot{W}^{2,1}$, whether we can produce a global weak solution is still open.

The outline of this paper is following. In section 2, we prove the global well-posedness of approximate problem. In section 3, we prove the existence of entropy weak solutions by a compactness argument. The uniqueness and stability results are proved in section 4 .

\section{Global wellposedness of the approximate equation}

We will prove the existence of solutions by using the viscous approximation. Consider the parabolic equation

$$
m_{\varepsilon, t}+\left[\left(u_{\varepsilon}^{2}-u_{\varepsilon, x}^{2}\right) m_{\varepsilon}\right]_{x}=\varepsilon m_{\varepsilon, x x} .
$$


It can be written as

$$
u_{\varepsilon, t}-u_{\varepsilon, t x x}+\left[\left(u_{\varepsilon}^{2}-u_{\varepsilon, x}^{2}\right)\left(u_{\varepsilon}-u_{\varepsilon, x x}\right)\right]_{x}=\varepsilon u_{\varepsilon, x x}-\varepsilon u_{\varepsilon, x x x x},
$$

or

$$
u_{\varepsilon, t}+u_{\varepsilon}^{2} u_{\varepsilon, x}=\frac{1}{3} u_{\varepsilon, x}^{3}-\Phi\left(u_{\varepsilon}\right)+\varepsilon u_{\varepsilon, x x} .
$$

Rewrite the equation as

$$
u_{\varepsilon, t}-\varepsilon u_{\varepsilon, x x}=-u_{\varepsilon}^{2} u_{\varepsilon, x}+\frac{1}{3} u_{\varepsilon, x}^{3}-\Phi\left(u_{\varepsilon}\right),
$$

and denote the right-hand-side as $F\left(u_{\varepsilon}, u_{\varepsilon, x}\right)$ for short.

The initial data is the smooth approximation of the original initial data:

$$
u_{\varepsilon}(0, x)=u_{0 \varepsilon}(x)=J_{\varepsilon} * u_{0},
$$

where

$$
J_{\varepsilon}(x)=\frac{C}{\varepsilon} \exp \left(\varepsilon^{2} /\left(x^{2}+\varepsilon^{2}\right)\right), \quad C \text { is a constant such that } \int_{\mathbb{R}} J_{\varepsilon}(x) d x=1 .
$$

In this section, we assume $\varepsilon>0$ fixed.

We prove global wellposedness by two steps. First, we prove local wellposedness. Then we prove global wellposedness by a global a priori estimate.

\subsection{Local well-posedness}

Proposition 2.1 (Local wellposedness). There exists a time $T_{0}>0$, such that the Cauchy problem (2.19), (2.20) is well-posed, $u \in C\left(\left[0, T_{0}\right] ; H^{2}(\mathbb{R})\right)$.

The proof of local wellposedness is standard. We record it here for completeness.

Proof. We first consider the linearized equation

$$
u_{\varepsilon, t}-\varepsilon u_{\varepsilon, x x}=-\bar{u}_{\varepsilon}^{2} \bar{u}_{\varepsilon, x}+\frac{1}{3} \bar{u}_{\varepsilon, x}^{3}-\Phi\left(\bar{u}_{\varepsilon}\right),
$$

or

$$
m_{\varepsilon, t}-\varepsilon m_{\varepsilon, x x}=\left[\left(-\bar{u}_{\varepsilon}^{2}+\bar{u}_{\varepsilon, x}^{2}\right) \bar{m}_{\varepsilon}\right]_{x}
$$

where $\bar{m}_{\varepsilon}=\bar{u}_{\varepsilon}-\bar{u}_{\varepsilon, x x}$.

By Duhamel principle, we can express the solution by the integral form,

$$
u_{\varepsilon}(t, x)=S(t) u_{0 \varepsilon}+\int_{0}^{t} S(t-s)\left(-\bar{u}_{\varepsilon}^{2} \bar{u}_{\varepsilon, x}+\frac{1}{3} \bar{u}_{\varepsilon, x}^{3}-\Phi\left(\bar{u}_{\varepsilon}\right)\right)(s, x) d s
$$

where $S(t) v=\frac{1}{\sqrt{4 \pi \varepsilon t}} e^{-\frac{x^{2}}{2 \pi \varepsilon t}} * v$ is the heat semigroup.

Define a map $\mathcal{T}$ as

$$
\mathcal{T} u_{\varepsilon}=S(t) u_{0 \varepsilon}+\int_{0}^{t} S(t-s)\left(-u_{\varepsilon}^{2} u_{\varepsilon, x}+\frac{1}{3} u_{\varepsilon, x}^{3}-\Phi\left(u_{\varepsilon}\right)\right)(s, x) d s,
$$

then (2.24) is equivalent to $u_{\varepsilon}=\mathcal{T} \bar{u}_{\varepsilon}$. In the following, we will show that for $T$ sufficiently small, $\mathcal{T}$ is a contraction mapping from $L^{\infty}\left(0, T_{0} ; H^{2}(\mathbb{R})\right)$ to itself. 
(i) $\mathcal{T}$ is a map from $L^{\infty}\left(0, T_{0} ; H^{2}(\mathbb{R})\right)$ to itself. Suppose $u_{\varepsilon} \in L^{\infty}\left(0, T_{0} ; H^{2}(\mathbb{R})\right)$. We prove $\mathcal{T} u_{\varepsilon} \in L^{\infty}\left(0, T_{0} ; H^{2}(\mathbb{R})\right)$, where $T_{0}$ is a positive number to be determined later. In fact,

$$
\begin{aligned}
& \left\|\partial_{x}^{2} \mathcal{T} u_{\varepsilon}(t, x)\right\|_{L_{T_{0}}^{\infty} L_{x}^{2}} \\
\leq & \left\|\partial_{x}^{2} S(t) u_{0 \varepsilon}\right\|_{L_{T_{0}}^{\infty} L_{x}^{2}}+\left\|\int_{0}^{t} \int_{\mathbb{R}} \frac{1}{\sqrt{4 \pi \varepsilon(t-s)}} \partial_{x} e^{-\frac{(x-y)^{2}}{2 \pi \varepsilon(t-s)}} \partial_{y}\left(-u_{\varepsilon}^{2} u_{\varepsilon, x}+\frac{1}{3} u_{\varepsilon, x}^{3}-\Phi\left(u_{\varepsilon}\right)\right)(s, y) d y d s\right\|_{L_{T_{0}}^{\infty} L_{x}^{2}} \\
\leq & \left\|\partial_{x}^{2} u_{0 \varepsilon}\right\|_{L_{x}^{2}}+\left\|\int_{0}^{t} \frac{1}{\sqrt{4 \pi \varepsilon(t-s)}}\right\| \partial_{x} e^{-\frac{x^{2}}{2 \pi \varepsilon(t-s)}}\left\|_{L_{x}^{1}} \cdot\right\|\left(-u_{\varepsilon}^{2} u_{\varepsilon, x}+\frac{1}{3} u_{\varepsilon, x}^{3}-\Phi\left(u_{\varepsilon}\right)\right)_{x}(s, \cdot)\left\|_{L_{x}^{2}} d s\right\|_{L_{T_{0}}^{\infty}} \\
\leq & \left\|\partial_{x}^{2} u_{0 \varepsilon}\right\|_{L_{x}^{2}}+\frac{C}{\sqrt{\varepsilon}} \sqrt{T_{0}}\left(\left\|u_{\varepsilon}\right\|_{L_{T_{0}}^{\infty} H_{x}^{2}}^{3}+\|\Phi\|_{L_{T_{0}}^{\infty} L_{x}^{2}}\right) .
\end{aligned}
$$

Here and in the following, we use $C$ to express an absolute constant which may be different from line by line.

By considering the convolution expression of $\Phi$ and Young's inequality, we have

$$
\begin{aligned}
\|\Phi\|_{L_{T_{0}}^{\infty} L_{x}^{2}} & \leq\left\|\frac{1}{2} e^{-|x|}\right\|_{L_{x}^{1}} \cdot\left\|\frac{4}{3} u_{\varepsilon, x}^{3}+2 u_{\varepsilon}^{2} u_{\varepsilon, x}+2 u_{\varepsilon} u_{\varepsilon, x} u_{\varepsilon, x x}\right\|_{L_{T_{0}}^{\infty} L_{x}^{2}} \\
& \leq C\left\|u_{\varepsilon}\right\|_{L_{T}^{\infty} H_{x}^{2}}^{3}
\end{aligned}
$$

So $\partial_{x}^{3} \mathcal{T} u_{\varepsilon}(t, x) \in L^{\infty}\left(0, T ; L^{2}(\mathbb{R})\right)$.

$$
\begin{aligned}
& \left\|\mathcal{T} u_{\varepsilon}(t, x)\right\|_{L_{T_{0}}^{\infty} L_{x}^{2}} \\
\leq & \left\|S(t) u_{0 \varepsilon}\right\|_{L_{T_{0}}^{\infty} L_{x}^{2}}+\left\|\int_{0}^{t} \int_{\mathbb{R}} \frac{1}{\sqrt{4 \pi \varepsilon(t-s)}} e^{-\frac{(x-y)^{2}}{2 \pi \varepsilon(t-s)}}\left(-u_{\varepsilon}^{2} u_{\varepsilon, x}+\frac{1}{3} u_{\varepsilon, x}^{3}-\Phi\left(u_{\varepsilon}\right)\right)(s, y) d y d s\right\|_{L_{T_{0}}^{\infty} L_{x}^{2}} \\
\leq & \left\|u_{0 \varepsilon}\right\|_{L_{x}^{2}}+\left\|\int_{0}^{t} \frac{1}{\sqrt{4 \pi \varepsilon(t-s)}}\right\| e^{-\frac{x^{2}}{2 \pi \varepsilon(t-s)}}\left\|_{L_{x}^{1}} \cdot\right\|-u_{\varepsilon}^{2} u_{\varepsilon, x}+\frac{1}{3} u_{\varepsilon, x}^{3}-\Phi\left(u_{\varepsilon}\right)(s, \cdot)\left\|_{L_{x}^{2}} d s\right\|_{L_{T_{0}}^{\infty}} \\
\leq & \left\|u_{0 \varepsilon}\right\|_{L_{x}^{2}}+\frac{C}{\sqrt{\varepsilon}} T_{0}\left(\left\|u_{\varepsilon}\right\|_{L_{T_{0}}^{\infty} H_{x}^{2}}^{3}+\|\Phi\|_{L_{T_{0}}^{\infty} L_{x}^{2}}\right) .
\end{aligned}
$$

So we can conclude that $\mathcal{T} u_{\varepsilon} \in L^{\infty}\left(0, T_{0} ; H^{2}(\mathbb{R})\right)$ by interpolation. 
(ii) When $T_{0}$ is sufficiently small, $\mathcal{T}$ is a contraction mapping.

$$
\begin{aligned}
& \|\mathcal{T} u-\mathcal{T} v\|_{L_{T_{0}}^{\infty} H_{x}^{2}} \\
& \leq C\left\|\int_{0}^{t} \int_{\mathbb{R}} \frac{\partial_{x} e^{-\frac{(x-y)^{2}}{2 \pi \varepsilon(t-s)}}}{\sqrt{4 \pi \varepsilon(t-s)}} \partial_{y}\left[-u_{\varepsilon}^{2} u_{\varepsilon, x}+\frac{1}{3} u_{\varepsilon, x}^{3}-\Phi\left(u_{\varepsilon}\right)-\left(-v_{\varepsilon}^{2} v_{\varepsilon, x}+\frac{1}{3} v_{\varepsilon, x}^{3}-\Phi\left(v_{\varepsilon}\right)\right)\right](s, y) d y d s\right\|_{L_{T_{0}}^{\infty} L_{x}^{2}} \\
& +C\left\|\int_{0}^{t} \int_{\mathbb{R}} \frac{e^{-\frac{(x-y)^{2}}{2 \pi \varepsilon(t-s)}}}{\sqrt{4 \pi \varepsilon(t-s)}}\left[-u_{\varepsilon}^{2} u_{\varepsilon, x}+\frac{1}{3} u_{\varepsilon, x}^{3}-\Phi\left(u_{\varepsilon}\right)-\left(-v_{\varepsilon}^{2} v_{\varepsilon, x}+\frac{1}{3} v_{\varepsilon, x}^{3}-\Phi\left(v_{\varepsilon}\right)\right)\right](s, y) d y d s\right\|_{L_{T_{0}}^{\infty} L_{x}^{2}}
\end{aligned}
$$

By Young's inequality, the first term

$$
\begin{aligned}
& \left\|\int_{0}^{t} \int_{\mathbb{R}} \frac{\partial_{x} e^{-\frac{(x-y)^{2}}{2 \pi \varepsilon(t-s)}}}{\sqrt{4 \pi \varepsilon(t-s)}} \partial_{y}\left[-u_{\varepsilon}^{2} u_{\varepsilon, x}+\frac{1}{3} u_{\varepsilon, x}^{3}-\Phi\left(u_{\varepsilon}\right)-\left(-v_{\varepsilon}^{2} v_{\varepsilon, x}+\frac{1}{3} v_{\varepsilon, x}^{3}-\Phi\left(v_{\varepsilon}\right)\right)\right](s, y) d y d s\right\|_{L_{T_{0}}^{\infty} L_{x}^{2}} \\
\leq & \int_{0}^{t}\left\|\frac{\partial_{x} e^{-\frac{x^{2}}{2 \pi \varepsilon(t-s)}}}{\sqrt{4 \pi \varepsilon(t-s)}}\right\|_{L_{x}^{1}}\left\|\partial_{x}\left[-u_{\varepsilon}^{2} u_{\varepsilon, x}+\frac{1}{3} u_{\varepsilon, x}^{3}-\Phi\left(u_{\varepsilon}\right)-\left(-v_{\varepsilon}^{2} v_{\varepsilon, x}+\frac{1}{3} v_{\varepsilon, x}^{3}-\Phi\left(v_{\varepsilon}\right)\right)\right](s, x)\right\|_{L_{x}^{2}} d s \\
\leq & \int_{0}^{t} \frac{C}{\sqrt{\varepsilon s}} d s\left(\|u\|_{L_{T_{0}}^{\infty} H_{x}^{2}}+\|v\|_{L_{T_{0}}^{\infty} H_{x}^{2}}\right)\|u-v\|_{L_{T_{0}}^{\infty} H_{x}^{2}} \\
\leq & \frac{C}{\sqrt{\varepsilon}} \sqrt{T_{0}}\left(\|u\|_{L_{T_{0}}^{\infty} H_{x}^{2}}+\|v\|_{L_{T_{0}}^{\infty} H_{x}^{2}}\right)\|u-v\|_{L_{T_{0}}^{\infty} H_{x}^{2} .}
\end{aligned}
$$

The second term

$$
\begin{aligned}
& \left\|\int_{0}^{t} \int_{\mathbb{R}} \frac{e^{-\frac{(x-y)^{2}}{2 \pi \varepsilon(t-s)}}}{\sqrt{4 \pi \varepsilon(t-s)}}\left[-u_{\varepsilon}^{2} u_{\varepsilon, x}+\frac{1}{3} u_{\varepsilon, x}^{3}-\Phi\left(u_{\varepsilon}\right)-\left(-v_{\varepsilon}^{2} v_{\varepsilon, x}+\frac{1}{3} v_{\varepsilon, x}^{3}-\Phi\left(v_{\varepsilon}\right)\right)\right](s, y) d y d s\right\|_{L_{T_{0}}^{\infty} L_{x}^{2}} \\
\leq & \int_{0}^{t}\left\|\frac{e^{-\frac{x^{2}}{2 \pi \varepsilon(t-s)}}}{\sqrt{4 \pi \varepsilon(t-s)}}\right\|_{L_{x}^{1}}\left\|\left[-u_{\varepsilon}^{2} u_{\varepsilon, x}+\frac{1}{3} u_{\varepsilon, x}^{3}-\Phi\left(u_{\varepsilon}\right)-\left(-v_{\varepsilon}^{2} v_{\varepsilon, x}+\frac{1}{3} v_{\varepsilon, x}^{3}-\Phi\left(v_{\varepsilon}\right)\right)\right](s, x)\right\|_{L_{x}^{2}} d s \\
\leq & C T_{0}\left(\|u\|_{L_{T_{0}}^{\infty} H_{x}^{2}}+\|v\|_{L_{T_{0}}^{\infty} H_{x}^{2}}\right)\|u-v\|_{L_{T_{0}}^{\infty} H_{x}^{1}}
\end{aligned}
$$

When $T_{0}$ is small enough, we have

$$
\|\mathcal{T} u-\mathcal{T} v\|_{L_{T_{0}}^{\infty} H_{x}^{2}} \leq \frac{C}{\sqrt{\varepsilon}} \sqrt{T_{0}}\left(\|u\|_{L_{T_{0}}^{\infty} H_{x}^{2}}+\|v\|_{L_{T_{0}}^{\infty} H_{x}^{2}}\right)\|u-v\|_{L_{T_{0}}^{\infty} H_{x}^{2}} .
$$

When $T_{0}$ is small enough, we can obtain that the map $\mathcal{T}$ is a contraction mapping. By contraction mapping theorem, there is a unique fixed point of the map $\mathcal{T}$. So we proved the local wellposedness. 


\section{$2.2 \quad$ A priori estimates}

1. $L^{1}$ estimate of $m_{\varepsilon}$. Assume $\eta(v)=|v|, \eta_{\delta}(v)=J_{\delta}(v) * \eta(v)$, so $\eta_{\delta}(v) \rightarrow \eta(v)$ as $\delta \rightarrow 0$. Multiply equation (2.16) with $\eta_{\delta}^{\prime}\left(m_{\varepsilon}\right)$, take integral and let $\delta \rightarrow 0$,

$$
\int_{\mathbb{R}} \eta\left(m_{\varepsilon}(t, x)\right) d x-\int_{\mathbb{R}} \eta\left(m_{\varepsilon}(0, x)\right) d x \leq \varepsilon \int_{0}^{t} \int_{\mathbb{R}}-\eta^{\prime \prime}\left(m_{\varepsilon}\right) m_{\varepsilon, x}^{2} d x \leq 0 .
$$

So we have

$$
\left\|m_{\varepsilon}(t, \cdot)\right\|_{L^{1}} \leq\left\|m_{\varepsilon, 0}\right\|_{L^{1}}
$$

2. $H^{1}$ estimate. We have

$$
\begin{gathered}
\left(u_{\varepsilon}^{2}\right)_{t}+\left(\frac{1}{2} u_{\varepsilon}^{4}\right)_{x}=\frac{2}{3} u_{\varepsilon, x}^{3} u_{\varepsilon}-2 u_{\varepsilon} \Phi\left(u_{\varepsilon}\right)+2 \varepsilon u_{\varepsilon, x x} u_{\varepsilon} \\
\left(u_{\varepsilon, x}^{2}\right)_{t}+\left(u_{\varepsilon}^{2} u_{\varepsilon, x}^{2}\right)_{x}=2 u_{\varepsilon, x}^{3} u_{\varepsilon, x x}+\frac{4}{3} u_{\varepsilon}^{3} u_{\varepsilon, x}-2 u_{\varepsilon, x} \partial_{x}\left(1-\partial_{x}^{2}\right)^{-1}\left(\frac{1}{3} u_{\varepsilon, x}^{3}\right) \\
-2 u_{\varepsilon, x}\left(1-\partial_{x}^{2}\right)^{-1}\left[\frac{2}{3} u_{\varepsilon}^{3}+u u_{\varepsilon, x}^{2}\right]+2 \varepsilon u_{\varepsilon, x} u_{\varepsilon, x x x} .
\end{gathered}
$$

Take the summation and then integrate,

$$
\frac{d}{d t} \int_{\mathbb{R}} u_{\varepsilon}^{2}+u_{\varepsilon, x}^{2} d x=-2 \varepsilon \int_{\mathbb{R}} u_{\varepsilon, x}^{2}+u_{\varepsilon, x x}^{2} d x
$$

So we have the energy estimate,

$$
\int_{\mathbb{R}} u_{\varepsilon}^{2}(t, x)+u_{\varepsilon, x}^{2}(t, x) d x+2 \varepsilon \int_{0}^{t} \int_{\mathbb{R}} u_{\varepsilon, x}^{2}+u_{\varepsilon, x x}^{2} d x d t=\int_{\mathbb{R}} u_{\varepsilon}^{2}(0, x)+u_{\varepsilon, x}^{2}(0, x) d x .
$$

3. $L^{1}$ estimate of $u_{\varepsilon}$ and $u_{\varepsilon, x x}$. Multiply $\eta_{\delta}^{\prime}\left(u_{\varepsilon}\right)$ to the equation of $u_{\varepsilon}$, take integration, and let $\delta \rightarrow 0$,

$$
\begin{aligned}
& \int_{\mathbb{R}} \eta\left(u_{\varepsilon}(t, x)\right) d x-\int_{\mathbb{R}} \eta\left(u_{\varepsilon}(0, x)\right) d x \\
\leq & \int_{0}^{t} \int_{\mathbb{R}} \frac{1}{3}\left|u_{\varepsilon, x}\right|^{3}+\left|\Phi\left(u_{\varepsilon}\right)\right| d x+\varepsilon \int_{\mathbb{R}} \eta^{\prime}\left(u_{\varepsilon}\right) u_{\varepsilon, x x} d x \\
\leq & \int_{0}^{t} \int_{\mathbb{R}} \frac{1}{3}\left|u_{\varepsilon, x}\right|^{3} d x+\int_{0}^{t} \int_{\mathbb{R}}\left|\left(e^{-|x|} * \frac{1}{3} u_{\varepsilon, x}^{3}\right)\right| d x+\int_{0}^{t} \int_{\mathbb{R}}\left|e^{-|x|} * \partial_{x}\left[\frac{2}{3} u_{\varepsilon}^{3}+u_{\varepsilon} u_{\varepsilon, x}^{2}\right]\right| d x \\
& -\varepsilon \int_{0}^{t} \int_{\mathbb{R}} \eta^{\prime \prime}\left(u_{\varepsilon}\right) u_{\varepsilon, x}^{2} d x \\
\leq & \int_{0}^{t}\left(2\left\|u_{\varepsilon}\right\|_{H^{1}}^{3}+\left\|u_{\varepsilon}\right\|_{H^{1}}^{2}\left\|u_{\varepsilon, x x}\right\|_{L^{1}}+\left\|u_{\varepsilon, x}\right\|_{L^{\infty}}\left\|u_{\varepsilon, x}\right\|_{L^{2}}^{2}+4\left\|u_{\varepsilon}\right\|_{H^{1}}^{3}\right) d t
\end{aligned}
$$

So

$$
\left\|u_{\varepsilon}(t, \cdot)\right\|_{L^{1}}-\left\|u_{\varepsilon}(0, \cdot)\right\|_{L^{1}} \leq \int_{0}^{t} 6\left\|u_{\varepsilon}\right\|_{H^{1}}^{3}+\left\|u_{\varepsilon}\right\|_{H^{1}}^{2}\left(\left\|u_{\varepsilon}\right\|_{L^{1}}+\left\|m_{\varepsilon}\right\|_{L^{1}}\right) d t
$$




$$
\left\|u_{\varepsilon}(t)\right\|_{L^{1}} \leq\left\|u_{\varepsilon, 0}\right\|_{L^{1}} e^{E_{0} t}+\left(6 E_{0}^{1 / 2}+\left\|m_{\varepsilon, 0}\right\|_{L^{1}}\right)\left(e^{E_{0} t}-1\right) .
$$

Combining the estimate of $m$, we obtain the $L^{1}$ estimate of $u_{x x}$

$$
\left\|u_{\varepsilon, x x}(t)\right\|_{L^{1}} \leq\left\|m_{\varepsilon, 0}\right\|_{L^{1}}+\left\|u_{\varepsilon, 0}\right\|_{L^{1}} e^{E_{0} t}+\left(6 E_{0}^{1 / 2}+\left\|m_{\varepsilon, 0}\right\|_{L^{1}}\right)\left(e^{E_{0} t}-1\right) .
$$

Above three estimates are all uniform with respect to $\varepsilon$. Next, we derive the high order energy estimates, which are depend on $\varepsilon$.

\section{4. $H^{2}$ estimate.}

$$
u_{\varepsilon, x x t}-\varepsilon u_{\varepsilon, x x x x}=\partial_{x}^{2} F\left(u_{\varepsilon}, u_{\varepsilon, x}\right),
$$

where the right-hand-side is

$$
\begin{aligned}
\partial_{x}^{2} F\left(u_{\varepsilon}, u_{\varepsilon, x}\right) & =\partial_{x}\left(-2 u_{\varepsilon} u_{\varepsilon, x}^{2}-u_{\varepsilon}^{2} u_{\varepsilon, x x}+u_{\varepsilon, x}^{2} u_{\varepsilon, x x}-\partial_{x} \Phi\left(u_{\varepsilon}\right)\right) \\
& =-6 u_{\varepsilon} u_{\varepsilon, x} u_{\varepsilon, x x x}-2 u_{\varepsilon, x}^{3}-u_{\varepsilon}^{2} u_{\varepsilon, x x x}+2 u_{\varepsilon, x} u_{\varepsilon, x x}^{2}+u_{\varepsilon, x}^{2} u_{\varepsilon, x x x}-\partial_{x}^{2} \Phi\left(u_{\varepsilon}\right) .
\end{aligned}
$$

Multiply $u_{\varepsilon, x x}$ and then take the integral,

$$
\begin{aligned}
\frac{d}{d t} \int_{\mathbb{R}} \frac{1}{2} u_{\varepsilon, x x}^{2} d x+\varepsilon \int_{\mathbb{R}} u_{\varepsilon, x x x}^{2} d x & \leq \int_{\mathbb{R}}\left|u_{\varepsilon, x x x}\left(-2 u_{\varepsilon} u_{\varepsilon, x}^{2}-u_{\varepsilon}^{2} u_{\varepsilon, x x}+u_{\varepsilon, x}^{2} u_{\varepsilon, x x}-\partial_{x} \Phi\left(u_{\varepsilon}\right)\right)\right| d x \\
& \leq \int_{\mathbb{R}} \frac{\varepsilon}{2} u_{\varepsilon, x x x}^{2}+\frac{2}{\varepsilon}\left(-2 u_{\varepsilon} u_{\varepsilon, x}^{2}-u_{\varepsilon}^{2} u_{\varepsilon, x x}+u_{\varepsilon, x}^{2} u_{\varepsilon, x x}-\partial_{x} \Phi\left(u_{\varepsilon}\right)\right)^{2} d x
\end{aligned}
$$

So we obtain

$$
\begin{aligned}
& \frac{d}{d t} \int_{\mathbb{R}} \frac{1}{2} u_{\varepsilon, x x}^{2} d x+\frac{\varepsilon}{2} \int_{\mathbb{R}} u_{\varepsilon, x x x}^{2} d x \\
\leq & \int_{\mathbb{R}} \frac{8}{\varepsilon}\left[4 u_{\varepsilon}^{2} u_{\varepsilon, x}^{4}+u_{\varepsilon}^{4} u_{\varepsilon, x x}^{2}+u_{\varepsilon, x}^{4} u_{\varepsilon, x x}^{2}+\left(\partial_{x} \Phi\right)^{2}\left(u_{\varepsilon}\right)\right] d x \\
\leq & \frac{32}{\varepsilon}\left(\left\|u_{\varepsilon}\right\|_{H^{1}}^{2}\left\|u_{\varepsilon, x x}\right\|_{L^{1}}^{2}\left\|u_{\varepsilon}\right\|_{H^{1}}^{2}+\left\|u_{\varepsilon}\right\|_{H^{1}}^{4}\left\|u_{\varepsilon}\right\|_{H^{2}}^{2}+\left\|u_{\varepsilon, x x}\right\|_{L^{1}}^{4}\left\|u_{\varepsilon}\right\|_{H^{2}}^{2}+\left\|\partial_{x} \Phi\left(u_{\varepsilon}\right)\right\|_{L^{2}}^{2}\right) .
\end{aligned}
$$$$
\text { Considering (1.4), we have }
$$$$
\begin{aligned}
\left\|\partial_{x} \Phi\left(u_{\varepsilon}\right)\right\|_{L^{2}}^{2} & \leq\left\|\frac{4}{3} u_{\varepsilon, x}^{3}+2 u_{\varepsilon}^{2} u_{\varepsilon, x}+2 u_{\varepsilon} u_{\varepsilon, x} u_{\varepsilon, x x}\right\|\left\|_{L_{x}^{2}}\right\| \frac{1}{2} e^{-|x|} \|_{L_{x}^{1}} \\
& \leq \frac{4}{3}\left\|u_{\varepsilon, x x}\right\|_{L_{x}^{1}}^{2}\left\|u_{\varepsilon}\right\|_{H_{x}^{1}}+2\left\|u_{\varepsilon}\right\|_{H_{x}^{1}}^{3}+2\left\|u_{\varepsilon}\right\|_{H_{x}^{1}}\left\|u_{\varepsilon, x x}\right\|_{L_{x}^{1}}\left\|u_{\varepsilon}\right\|_{H_{x}^{2}} \\
& \leq\left(\frac{4}{3}\left\|u_{\varepsilon, x x}\right\|_{L_{x}^{1}}^{2}+2\left\|u_{\varepsilon}\right\|_{H_{x}^{1}}^{2}+2\left\|u_{\varepsilon}\right\|_{H_{x}^{1}}\left\|u_{\varepsilon, x x}\right\|_{L_{x}^{1}}\right)\left\|u_{\varepsilon}\right\|_{H_{x}^{2}} .
\end{aligned}
$$

Therefore,

$$
\begin{aligned}
& \frac{d}{d t} \frac{1}{2}\left\|u_{\varepsilon}\right\|_{H_{x}^{2}}^{2}+\frac{\varepsilon}{2}\left\|u_{\varepsilon, x x x}(t, \cdot)\right\|_{L_{x}^{2}}^{2} \\
& \leq \frac{C}{\varepsilon}\left[\left\|u_{\varepsilon}\right\|_{H_{x}^{1}}^{4}\left\|u_{\varepsilon, x x}\right\|_{L_{x}^{1}}^{2}+\left\|u_{\varepsilon, x x}\right\|_{L_{x}^{1}}^{4}+\left\|u_{\varepsilon}\right\|_{H_{x}^{1}}^{4}+\left\|u_{\varepsilon}\right\|_{H_{x}^{1}}^{2}\left\|u_{\varepsilon, x x}\right\|_{L_{x}^{1}}^{2}\right. \\
&\left.\quad+\left\|u_{\varepsilon}\right\|_{H_{x}^{2}}^{2}\left(\left\|u_{\varepsilon}\right\|_{H_{x}^{1}}^{4}+\left\|u_{\varepsilon, x x}\right\|_{L_{x}^{1}}^{4}+1\right)\right] .
\end{aligned}
$$


By Gronwall inequality, we have for any fixed $T,\left\|u_{\varepsilon}\right\|_{L_{T}^{\infty} H_{x}^{2}}$ and $\left\|u_{\varepsilon, x}\right\|_{L_{T}^{2} H_{x}^{2}}$ are bounded by some constant $C(\varepsilon, T)$, which is related with the initial data, $\varepsilon$ and $T$.

5. $H^{\ell}$ estimate. We prove $H^{\ell}(\ell>2)$ estimate by a induction argument. Assume that for any $T, \varepsilon>0$, there is a constant $C(\varepsilon, T)$, such that $\left\|u_{\varepsilon}\right\|_{L_{T}^{\infty} H_{x}^{\ell-1}}+\left\|u_{\varepsilon}\right\|_{L_{T}^{2} H_{x}^{\ell}}<C(\varepsilon, T)$, for every $t \in[0, T]$. Then we derive $H^{\ell}$ estimate. Take $\ell$ th order derivative

$$
\begin{gathered}
\left(\partial_{x}^{\ell} u_{\varepsilon}\right)_{t}-\varepsilon \partial_{x}^{\ell} u_{\varepsilon, x x}=\partial_{x}^{\ell} F\left(u_{\varepsilon}, u_{\varepsilon, x}\right) . \\
\frac{d}{d t} \int_{\mathbb{R}} \frac{1}{2}\left|\partial_{x}^{\ell} u_{\varepsilon}\right|^{2} d x+\varepsilon \int_{\mathbb{R}}\left|\partial_{x}^{\ell+1} u_{\varepsilon}\right|^{2} d x=\int_{\mathbb{R}}\left|\partial_{x}^{\ell} F\left(u_{\varepsilon}, u_{\varepsilon, x}\right) \partial_{x}^{\ell} u_{\varepsilon}\right| d x \\
\leq \frac{2}{\varepsilon} \int_{\mathbb{R}}\left|\partial_{x}^{\ell-1} F\left(u_{\varepsilon}, u_{\varepsilon, x}\right)\right|^{2} d x+\frac{\varepsilon}{2} \int_{\mathbb{R}}\left|\partial_{x}^{\ell+1} u_{\varepsilon}\right|^{2} d x
\end{gathered}
$$

So

$$
\begin{aligned}
\frac{d}{d t} \int_{\mathbb{R}} \frac{1}{2}\left|\partial_{x}^{\ell} u_{\varepsilon}\right|^{2} d x+\frac{\varepsilon}{2} \int_{\mathbb{R}}\left|\partial_{x}^{\ell+1} u_{\varepsilon}\right|^{2} d x & \leq \frac{2}{\varepsilon}\left\|\partial_{x}^{\ell-1} F\left(u_{\varepsilon}, u_{\varepsilon, x}\right)\right\|_{L_{x}^{2}}^{2} \\
& \leq \frac{2}{\varepsilon}\left[\left\|u_{\varepsilon}^{2}+u_{\varepsilon, x}^{2}\right\|_{L_{x}^{\infty}}\left\|\partial_{x}^{\ell} u_{\varepsilon}\right\|_{L_{x}^{2}}^{2}+\left\|Q\left(u_{\varepsilon}, \ldots, \partial_{x}^{\ell-1} u_{\varepsilon}\right)\right\|_{L_{x}^{2}}^{2}\right]
\end{aligned}
$$

We only care about the term with $\partial_{x}^{\ell-1} u_{\varepsilon}$ in the term $Q$, since the lower order terms like $\partial_{x}^{i} u_{\varepsilon} \partial_{x}^{j} u_{\varepsilon} \partial_{x}^{k} u_{\varepsilon}$ with $i, j, k<\ell-1$ can be estimated by

$$
\left\|\partial_{x}^{i} u_{\varepsilon} \partial_{x}^{j} u_{\varepsilon} \partial_{x}^{k} u_{\varepsilon}\right\|_{L_{x}^{2}} \leq\left\|\partial_{x}^{i} u_{\varepsilon}\right\|_{L_{x}^{\infty}}\left\|\partial_{x}^{j} u_{\varepsilon}\right\|_{L_{x}^{\infty}}\left\|\partial_{x}^{k} u_{\varepsilon}\right\|_{L_{x}^{2}} \leq\left\|u_{\varepsilon}\right\|_{H_{x}^{\ell-1}}^{3}<[C(\varepsilon, T)]^{3} .
$$

When $\ell=3$, there is term $u_{\varepsilon, x} u_{\varepsilon, x x}^{2}$. To estimate this term, we need to use the boundedness of $\left\|u_{\varepsilon, x x x}\right\|_{L_{T}^{2} L_{x}^{2}}$.

$$
\begin{aligned}
\left\|u_{\varepsilon, x} u_{\varepsilon, x x}^{2}\right\|_{L_{T}^{2} L_{x}^{2}} & \leq\left\|u_{\varepsilon, x}\right\|_{L_{T}^{\infty} L_{x}^{\infty}}\left\|u_{\varepsilon, x x}\right\|_{L_{T}^{2} L_{x}^{\infty}}\left\|u_{\varepsilon, x x}\right\|_{L_{T}^{\infty} L_{x}^{2}} \\
& \leq\left\|u_{\varepsilon, x}\right\|_{L_{T}^{\infty} L_{x}^{\infty}}\left\|u_{\varepsilon, x x}\right\|_{L_{T}^{2} H_{x}^{1}}\left\|u_{\varepsilon, x x}\right\|_{L_{T}^{\infty} L_{x}^{2}}<\infty .
\end{aligned}
$$

When $\ell \geq 4, Q$ is linear with respect to $\partial_{x}^{\ell-1} u_{\varepsilon}$.

$\left\|\partial_{x}^{i} u_{\varepsilon} \partial_{x}^{j} u_{\varepsilon} \partial_{x}^{\ell-1} u_{\varepsilon}\right\|_{L_{t}^{\infty} L_{x}^{2}} \leq\left\|\partial_{x}^{i} u_{\varepsilon}\right\|_{L_{t}^{\infty} L_{x}^{\infty}}\left\|\partial_{x}^{j} u_{\varepsilon}\right\|_{L_{t}^{\infty} L_{x}^{\infty}}\left\|\partial_{x}^{\ell-1} u_{\varepsilon}\right\|_{L_{t}^{\infty} L_{x}^{2}} \leq\left\|u_{\varepsilon}\right\|_{L_{t}^{\infty} H_{x}^{\ell-1}}^{3}<[C(\varepsilon, T)]^{3}$.

So in every case, we can obtain $\left\|u_{\varepsilon}\right\|_{L_{T}^{\infty} H_{x}^{\ell}}+\left\|u_{\varepsilon}\right\|_{L_{T}^{2} H_{x}^{\ell+1}}$ is bounded.

\subsection{Global wellposedness and regularity}

Then from the above a priori estimates, we have

Proposition 2.2 (Global well-posedness and regularity). If $\varepsilon$ is a fixed positive constant. For any $T>0, \ell \geq 2$. If the initial data $u_{0 \varepsilon} \in H^{\ell}(\mathbb{R})$, then there is a unique solution $u_{\varepsilon}(t, x) \in$ $L^{\infty}\left(0, T ; H^{\ell}(\mathbb{R})\right) \cap L^{2}\left(0, T ; H^{\ell+1}(\mathbb{R})\right), u_{\varepsilon, t} \in L^{\infty}\left(0, T ; H^{\ell-2}(\mathbb{R})\right) \cap L^{2}\left(0, T ; H^{\ell-1}(\mathbb{R})\right)$. 


\section{Existence of entropy weak solutions to $(\mathrm{cCH})$}

Definition 3.1 (Weak solution). We call a function $u: \mathbb{R}_{+} \times \mathbb{R} \rightarrow \mathbb{R}$ a weak solution of the Cauchy problem (1.3), (1.5) if

(i) $u \in L^{\infty}\left(\mathbb{R}_{+} ; H^{1}(\mathbb{R}) \cap \dot{W}^{1,4}(\mathbb{R})\right)$, and

(ii) u satisfies (1.3), (1.5) in distributional sense, that is

$$
\iint_{\mathbb{R}_{+} \times \mathbb{R}} u \phi_{t}+\frac{1}{3} u^{3} \phi_{x}+\frac{1}{3} u_{x}^{3} \phi-\Phi(u) \phi d x d t+\int_{\mathbb{R}} u_{0}(x) \phi(0, x) d x=0,
$$

where

$$
\Phi(u)=\left(1-\partial_{x}^{2}\right)^{-1}\left[\frac{4}{3} u_{x}^{3}+2 u^{2} u_{x}+2 u u_{x} u_{x x}\right]=\frac{1}{2} e^{-|x|} *\left[\frac{4}{3} u_{x}^{3}+2 u^{2} u_{x}+2 u u_{x} u_{x x}\right] .
$$

Definition 3.2 (Entropy weak solution). We call a function $u: \mathbb{R}_{+} \times \mathbb{R} \rightarrow \mathbb{R}$ an entropy weak solution of the Cauchy problem (1.3), (1.5) provided

(i) $u$ is a weak solution in the sense of Definition 3.1,

(ii) $u \in L^{\infty}(0, T ; X)$ for any $T>0$, and

(iii) for any convex $C^{2}$ entropy $\eta: \mathbb{R} \rightarrow \mathbb{R}$ with corresponding entropy flux $q: \mathbb{R} \rightarrow \mathbb{R}$ defined by $q^{\prime}(u)=\eta^{\prime}(u) u^{2}$ there holds

$$
\partial_{t} \eta\left(u_{x}\right)+\partial_{x}\left(u^{2} \eta\left(u_{x}\right)-q\left(u_{x}\right)\right)+\eta^{\prime}\left(u_{x}\right) \partial_{x} \Phi(u)+2 u u_{x}\left(u_{x} \eta^{\prime}\left(u_{x}\right)-\eta\left(u_{x}\right)\right) \leq 0,
$$

that is, for any $\varphi \in C_{c}^{\infty}([0, \infty) \times \mathbb{R}), \varphi \geq 0$,

$$
\begin{aligned}
& \iint_{\mathbb{R}_{+} \times \mathbb{R}} \eta\left(u_{x}\right) \varphi_{t}+\left(u^{2} \eta\left(u_{x}\right)-q\left(u_{x}\right)\right) \varphi_{x}-\eta^{\prime}\left(u_{x}\right) \partial_{x} \Phi(u) \varphi-2 u u_{x}\left(u_{x} \eta^{\prime}\left(u_{x}\right)-\eta\left(u_{x}\right)\right) \varphi d x d t \\
& \quad+\int_{\mathbb{R}} \eta\left(u_{0}(x)\right) \varphi(0, x) d x \geq 0 .
\end{aligned}
$$

Our main theorem in this section is

Theorem 3.1 (Existence). Suppose the initial data $u_{0} \in X$. There exists at least one entropy weak solution to the Cauchy problem (1.3), (1.5).

Proof. We take a sequence of positive numbers $\left\{\varepsilon_{k}\right\}_{k=1}^{\infty}$ tending to zero. Then we obtain a sequence of solutions $\left\{u_{\varepsilon_{k}}\right\}_{k=1}^{\infty}$. From section 2.2, we know this sequence satisfies uniform $L^{1}, H^{1}, \dot{W}^{1, \infty}$, and $\dot{W}^{2,1}$ estimates.

So by Helly's theorem, as $k \rightarrow \infty$,

$$
u_{\varepsilon_{k}, x} \rightarrow u_{x} \quad \text { a.e. in } \mathbb{R}_{+} \times \mathbb{R},
$$

and hence

$$
u_{\varepsilon_{k}, x} \rightarrow u_{x} \quad \text { in } L_{l o c}^{p}\left(\mathbb{R}_{+} \times \mathbb{R}\right) \text { for all } p \in[1, \infty)
$$


By the $\dot{W}^{1, \infty}$ estimate and the energy estimate, there holds

$$
u_{\varepsilon_{k}, x} \rightarrow u_{x} \quad \text { in } L^{p}((0, T) \times \mathbb{R}) \text { for all } T>0 \text {, for all } p \in[1, \infty) .
$$

Further more, we can obtain that $u_{\varepsilon_{k}} \rightarrow u$ pointwise. So in order to prove (3.55), it suffices to prove the convergence of term $\Phi\left(u_{\varepsilon_{k}}\right)$. In fact,

$$
\begin{aligned}
& \left\|\Phi\left(u_{\varepsilon_{k}}\right)-\Phi(u)\right\|_{L^{p}} \\
= & \left\|\frac{1}{2} e^{-|x|} * \frac{1}{3}\left(u_{\varepsilon, x}^{3}-u_{x}^{3}\right)+\frac{1}{2}(\operatorname{sgn} x) e^{-|x|} *\left[\left(\frac{2}{3} u_{\varepsilon_{k}}^{3}+u_{\varepsilon_{k}} u_{\varepsilon_{k}, x}^{2}\right)-\left(\frac{2}{3} u^{3}+u u_{x}^{2}\right)\right]\right\|_{L^{p}} \\
\leq & 2\left(\left\|u_{\varepsilon_{k}, x}^{3}-u_{x}^{3}\right\|_{L^{p}}+\left\|u_{\varepsilon_{k}}^{3}-u^{3}\right\|_{L^{p}}+\left\|u_{\varepsilon_{k}} u_{\varepsilon_{k}, x}^{2}-u u_{x}^{2}\right\|_{L^{p}}\right) \rightarrow 0 .
\end{aligned}
$$

So the limit function $u$ is a weak solution to $(\mathrm{cCH})$. Then we shall prove the entropy inequality. Since for $\eta \in C^{2}$ with $\eta^{\prime \prime}>0$, the approximate solution $u_{\varepsilon}$ satisfies

$$
\begin{aligned}
\partial_{t} \eta\left(u_{\varepsilon, x}\right)+\left(u_{\varepsilon}^{2} \eta\left(u_{\varepsilon, x}\right)-q\left(u_{\varepsilon, x}\right)\right)_{x}+2 u_{\varepsilon} u_{\varepsilon, x}\left(u_{\varepsilon, x} \eta^{\prime}\left(u_{\varepsilon, x}\right)-\eta\left(u_{\varepsilon, x}\right)\right) & \\
& +\eta^{\prime}\left(u_{\varepsilon, x}\right) \partial_{x} \Phi\left(u_{\varepsilon}\right)=\varepsilon \eta^{\prime}\left(u_{\varepsilon, x}\right)\left(u_{\varepsilon, x}\right)_{x x} .
\end{aligned}
$$

Multiply a positive test function,

$$
\begin{aligned}
& -\iint_{\mathbb{R}_{+} \times \mathbb{R}} \eta\left(u_{\varepsilon, x}\right) \varphi_{t}+\left[u_{\varepsilon}^{2} \eta\left(u_{\varepsilon}\right)-q\left(u_{\varepsilon, x}\right)\right] \varphi_{x} d x d t-\int_{\mathbb{R}} \eta\left(u_{0}(x)\right) \varphi(0, x) d x \\
& +\iint_{\mathbb{R}_{+} \times \mathbb{R}} 2 u_{\varepsilon} u_{\varepsilon, x}\left(u_{\varepsilon, x} \eta^{\prime}\left(u_{\varepsilon, x}\right)-\eta\left(u_{\varepsilon, x}\right)\right) \varphi+\eta^{\prime}\left(u_{\varepsilon, x}\right) \partial_{x} \Phi\left(u_{\varepsilon}\right) d x d t \\
= & -\iint_{\mathbb{R}_{+} \times \mathbb{R}} \varepsilon \eta\left(u_{\varepsilon, x}\right)_{x} \varphi_{x} d x d t-\varepsilon \iint_{\mathbb{R}_{+} \times \mathbb{R}} \eta^{\prime \prime} u_{\varepsilon, x x}^{2} \varphi d x d t \\
\leq & -\iint_{\mathbb{R}_{+} \times \mathbb{R}} \varepsilon \eta^{\prime}\left(u_{\varepsilon, x}\right) u_{\varepsilon, x x} \varphi_{x} d x d t .
\end{aligned}
$$

Since $u_{\varepsilon, x x}(t, \cdot) \in L^{1}(\mathbb{R})$ and $\varphi$ is compact support, the right-hand-side approaches 0 as $\varepsilon \rightarrow 0$. So $u$ is an entropy weak solution.

\section{$4 \quad W^{1,1}$ stability and uniqueness}

Suppose $u$ and $v$ are two entropy weak solutions with initial data $u_{0}$ and $v_{0}$ respectively. We estimate $W^{1,1}$ norm of their difference.

$1\left(|u-v|_{L^{1}}\right.$ estimate). Since $u, v \in \dot{W}^{2,1}, u, v$ are Lipschitz functions. The derivatives $u_{x}, v_{x}$ exist almost everywhere. From the equation, we know $u_{t}, v_{t}$ are also well-defined. The equations for $u$ and $v$ are

$$
\begin{aligned}
& u_{t}+u^{2} u_{x}=\frac{1}{3} u_{x}^{3}-\Phi(u), \\
& v_{t}+v^{2} v_{x}=\frac{1}{3} v_{x}^{3}-\Phi(v) .
\end{aligned}
$$


Take their difference

$$
(u-v)_{t}+u^{2}(u-v)_{x}+(u+v)(u-v) v_{x}=\frac{1}{3}\left(u_{x}-v_{x}\right)\left(u_{x}^{2}+u_{x} v_{x}+v_{x}^{2}\right)-(\Phi(u)-\Phi(v)) .
$$

Multiply $\operatorname{sgn}(u-v)$ and then take integral,

$\frac{d}{d t} \int_{\mathbb{R}}|u-v| d x \leq \int_{\mathbb{R}}\left(\left|2 u u_{x}\right|+\left|v_{x}\right||u+v|\right)|u-v| d x+\frac{1}{3}\left|u_{x}-v_{x}\right|\left|u_{x}^{2}+u_{x} v_{x}+v_{x}^{2}\right|+|\Phi(u)-\Phi(v)| d x$.

By the expression of $\Phi$,

$$
\begin{aligned}
& \|\Phi(u)-\Phi(v)\|_{L_{x}^{1}} \\
\leq & \frac{1}{3}\left\|\frac{1}{2} e^{-|x|}\right\|_{L_{x}^{1}}\left\|u_{x}-v_{x}\right\|_{L_{x}^{1}}\left\|u_{x}^{2}+u_{x} v_{x}+v_{x}^{2}\right\|_{L_{x}^{\infty}} \\
& +\left\|\frac{1}{2} e^{-|x|}\right\|_{L_{x}^{1}}\left(\frac{2}{3}\|u-v\|_{L_{x}^{1}}\left\|u^{2}+u v+v^{2}\right\|_{L_{x}^{\infty}}+\|u-v\|_{L_{x}^{1}}\left\|u_{x}^{2}\right\|_{L_{x}^{\infty}}+\left\|v\left(u_{x}+v_{x}\right)\right\|_{L_{x}^{\infty}}\left\|u_{x}-v_{x}\right\|_{L_{x}^{1}}\right)
\end{aligned}
$$

Considering the boundedness of the $\dot{W}^{2,1}$-norm of $u, v$, we have

$$
\frac{d}{d t}\|(u-v)(t, \cdot)\|_{L_{x}^{1}} \leq K\left(T,\left\|u_{0}\right\|_{H_{x}^{1}},\left\|v_{0}\right\|_{H_{x}^{1}},\left\|u_{0}\right\|_{\dot{W}_{x}^{2,1}},\left\|v_{0}\right\|_{\dot{W}_{x}^{2,1}}\right)\|(u-v)(t, \cdot)\|_{W_{x}^{1,1}}
$$

where $K$ is a constant related with $T,\left\|u_{0}\right\|_{H_{x}^{1}},\left\|v_{0}\right\|_{H_{x}^{1}},\left\|u_{0}\right\|_{\dot{W}_{x}^{2,1}},\left\|v_{0}\right\|_{\dot{W}_{x}^{2,1}}$.

$2\left(\left|u_{x}-v_{x}\right|_{L^{1}}\right.$ estimate). Denote $\eta_{k}(u)=|u-k|$. Consider the equation

$$
\left(u_{x}\right)_{t}+\left(u^{2} u_{x}-\frac{1}{3} u_{x}^{3}\right)_{x}+\Phi_{x}=0 .
$$

Multiply $\eta_{k}^{\prime}\left(u_{x}\right)$,

$$
\begin{gathered}
\left|u_{x}-k\right|_{t}+\operatorname{sgn}\left(u_{x}-k\right)\left(u^{2} u_{x}-\frac{1}{3} u_{x}^{3}\right)_{x}+\operatorname{sgn}\left(u_{x}-k\right) \partial_{x} \Phi=0 . \\
\iint_{\mathbb{R}_{+} \times \mathbb{R}}\left|u_{x}-k\right| \varphi_{t}+\operatorname{sgn}\left(u_{x}-k\right)\left(u^{2} u_{x}-\frac{1}{3} u_{x}^{3}-u^{2} k+\frac{1}{3} k^{3}\right) \varphi_{x}-\operatorname{sgn}\left(u_{x}-k\right)\left(\partial_{x} \Phi(u)+2 k u u_{x}\right) \varphi d x d t \geq 0 . \\
\iint_{\mathbb{R}_{+} \times \mathbb{R}}\left|u_{x}-v_{y}\right| \varphi_{t}+\operatorname{sgn}\left(u_{x}-v_{y}\right)\left(u^{2} u_{x}-\frac{1}{3} u_{x}^{3}-u^{2} v_{y}+\frac{1}{3} v_{y}^{3}\right) \varphi_{x}-\operatorname{sgn}\left(u_{x}-v_{y}\right)\left(\partial_{x} \Phi(u)+2 v_{y} u u_{x}\right) \varphi d x d t \geq 0 .
\end{gathered}
$$

Similarly, for $v$,

$$
\begin{aligned}
& \iint_{\mathbb{R}+\times \mathbb{R}}\left|v_{y}-k\right| \varphi_{s}+\operatorname{sgn}\left(v_{y}-k\right)\left(v^{2} v_{y}-\frac{1}{3} v_{y}^{3}-v^{2} k+\frac{1}{3} k^{3}\right) \varphi_{y}-\operatorname{sgn}\left(v_{y}-k\right)\left(\partial_{y} \Phi(v)+2 k v v_{y}\right) \varphi d y d s \geq 0 . \\
& \iint_{\mathbb{R}_{+} \times \mathbb{R}}\left|v_{y}-u_{x}\right| \varphi_{s}+\operatorname{sgn}\left(v_{y}-u_{x}\right)\left(v^{2} v_{y}-\frac{1}{3} v_{y}^{3}-v^{2} u_{x}+\frac{1}{3} u_{x}^{3}\right) \varphi_{y}-\operatorname{sgn}\left(v_{y}-u_{x}\right)\left(\partial_{y} \Phi(v)+2 u_{x} v v_{y}\right) \varphi d y d s \geq 0 .
\end{aligned}
$$


Combine (4.72) and (4.74),

$$
\begin{aligned}
& \iiint \int_{\mathbb{R}_{+}^{2} \times \mathbb{R}^{2}}\left|u_{x}-v_{y}\right|\left(\varphi_{t}+\varphi_{s}\right)+\operatorname{sgn}\left(u_{x}-v_{y}\right)\left[u^{2}\left(u_{x}-v_{y}\right)-\frac{1}{3}\left(u_{x}^{3}-v_{y}^{3}\right)\right] \varphi_{x} \\
& \quad-\operatorname{sgn}\left(u_{x}-v_{y}\right)\left[-v^{2}\left(u_{x}-v_{y}\right)+\frac{1}{3}\left(u_{x}^{3}-v_{y}^{3}\right)\right] \varphi_{y} \\
& \quad-\operatorname{sgn}\left(u_{x}-v_{y}\right)\left(\partial_{x} \Phi(u)-\partial_{y} \Phi(v)+2 v_{y} u u_{x}-2 u_{x} v v_{y}\right) \varphi d x d y d s d t \geq 0 .
\end{aligned}
$$

Take $\varphi(t, x, s, y)=\psi\left(\frac{t+s}{2}, \frac{x-y}{2}\right) \delta_{h}\left(\frac{t-s}{2}\right) \delta_{h}\left(\frac{x-y}{2}\right)$,

$$
\begin{gathered}
\iint_{\mathbb{R}_{+} \times \mathbb{R}}\left|u_{x}-v_{x}\right| \psi_{t}(t, x)+\operatorname{sgn}\left(u_{x}-v_{x}\right)\left[\left(u^{2}+v^{2}\right)\left(u_{x}-v_{x}\right)-\frac{2}{3}\left(u_{x}^{3}-v_{x}^{3}\right)\right] \psi_{x}(t, x) \\
-\operatorname{sgn}\left(u_{x}-v_{x}\right)\left(\partial_{x} \Phi(u)-\partial_{x} \Phi(v)+2 u_{x} v_{x}(u-v)\right) \psi(t, x) d x d t \geq 0 . \\
\iint_{\mathbb{R}_{+} \times \mathbb{R}}\left|u_{x}-v_{x}\right| \psi_{t}(t, x)+\left|u_{x}-v_{x}\right|\left[\left(u^{2}+v^{2}\right)-\frac{2}{3}\left(u_{x}^{2}+u_{x} v_{x}+v_{x}^{2}\right)\right] \psi_{x}(t, x) \\
-\operatorname{sgn}\left(u_{x}-v_{x}\right)\left(\partial_{x} \Phi(u)-\partial_{x} \Phi(v)+2 u_{x} v_{x}(u-v)\right) \psi(t, x) d x d t \geq 0 .
\end{gathered}
$$

By the $\dot{W}^{2,1}$ estimate and the energy estimate, we can find a constant $L$ such that

$$
\left|\left(u^{2}+v^{2}\right)-\frac{2}{3}\left(u_{x}^{2}+u_{x} v_{x}+v_{x}^{2}\right)\right|<L .
$$

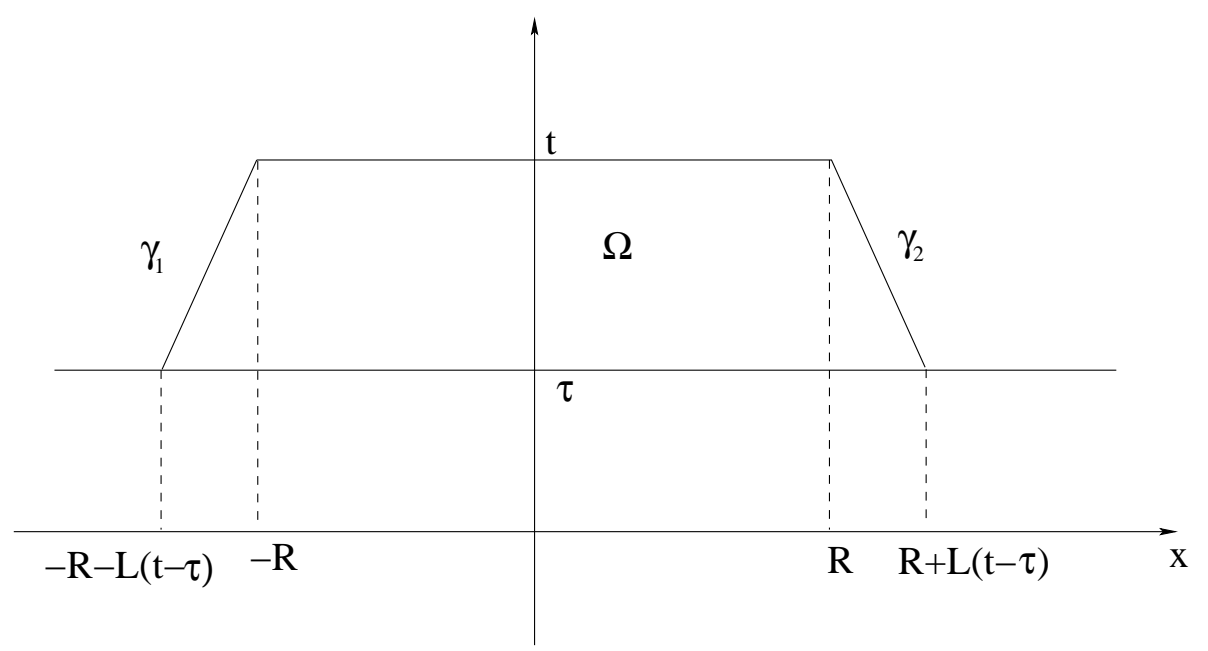

Figure 1: The domain in consideration.

Consider a trapezoid $\Omega$ as in Figure 1. The straight line $\gamma_{1}$ and $\gamma_{2}$ have slopes as $-L$ and $L$ separately.

Then we take $\psi$ to be a smooth approximation to the characteristic function of the trapezoid 
$\Omega$. We obtain

$$
\begin{aligned}
\int_{-R}^{R}\left|u_{x}(t, x)-v_{x}(t, x)\right| d x \leq & \int_{-R-L(t-\tau)}^{R+L(t-\tau)}\left|u_{x}(\tau, x)-v_{x}(\tau, x)\right| d x \\
& +\iint_{\Omega}\left|\partial_{x} \Phi(u)-\partial_{x} \Phi(v)+2 u_{x} v_{x}(u-v)\right| d x d t
\end{aligned}
$$

For the source term, we have the following estimate

$$
\begin{aligned}
& \left|\partial_{x} \Phi(u)-\partial_{x} \Phi(v)+2 u_{x} v_{x}(u-v)\right| \\
= & \mid \partial_{x}\left(1-\partial_{x}^{2}\right)^{-1}\left[\frac{1}{3}\left(u_{x}-v_{x}\right)\left(u_{x}^{2}+v_{x}^{2}+u_{x} v_{x}\right)\right]+\left(1-\partial_{x}^{2}\right)^{-1}\left[\frac{2}{3}(u-v)\left(u^{2}+u v+v^{2}\right)\right. \\
& \left.+u\left(u_{x}-v_{x}\right)\left(u_{x}+v_{x}\right)+(u-v) v_{x}^{2}\right]-\frac{2}{3}(u-v)\left(u^{2}+u v+v^{2}\right)-(u-v) u_{x}^{2} \\
& -v\left(u_{x}-v_{x}\right)\left(u_{x}+v_{x}\right)+2 u_{x} v_{x}(u-v) \mid .
\end{aligned}
$$

Take integral,

$$
\begin{aligned}
& \int_{\mathbb{R}}\left|\partial_{x} \Phi(u)-\partial_{x} \Phi(v)+2 u_{x} v_{x}(u-v)\right| d x \\
\leq & \sup _{t \in[0, T]}\left(\left\|\frac{1}{3}\left(u_{x}^{2}+v_{x}^{2}+u_{x} v_{x}\right)\right\|_{L^{\infty}}+\left\|\frac{2}{3}\left(u^{2}+u v+v^{2}\right)\right\|_{\mathbf{L}^{\infty}}+\left\|u_{x}^{2}\right\|_{L^{\infty}}+\left\|u\left(u_{x}+v_{x}\right)\right\|_{L^{\infty}}\right. \\
& \left.+2\left\|u_{x} v_{x}\right\|_{L^{\infty}}\right)\|u-v\|_{W^{1,1}}
\end{aligned}
$$

We let $R \rightarrow+\infty$ in (4.79), and add (4.68)

$$
\|u(t, \cdot)-v(t, \cdot)\|_{W^{1,1}} \leq\|u(0, \cdot)-v(0, \cdot)\|_{W^{1,1}}+\int_{0}^{t} M_{T}\|u(s, \cdot)-v(s, \cdot)\|_{W^{1,1}} d s,
$$

where $M_{T}$ is a constant of order $O\left(e^{C T}\right)$, and related with $\left\|u_{0}\right\|_{H^{1} \cap \dot{W}^{2,1}}$ and $\left\|v_{0}\right\|_{H^{1} \cap \dot{W}^{2,1}}$. By Gronwall's inequality, we obtain

$$
\|u(t, \cdot)-v(t, \cdot)\|_{W^{1,1}} \leq e^{M_{T} t}\|u(0, \cdot)-v(0, \cdot)\|_{W^{1,1}} .
$$

Acknowledgement. Q. Zhang would like to thank Prof. Alberto Bressan for helpful suggestion, and the anonymous reviewer for generous comments. This work is partially supported by NSF grant DMS-1411786.

\section{References}

[1] A. Bressan, G. Chen and Q. Zhang, Uniqueness of conservative solutions to the CamassaHolm equation via characteristics. Discr. Cont. Dyn. Syst. 35 (2015), 25-42. 
[2] A. Bressan and A. Constantin, Global conservative solutions to the Camassa-Holm equation, Arch. Rat. Mech. Anal. 183 (2007), 215-239.

[3] A. Bressan and A. Constantin, Global dissipative solutions to the Camassa-Holm equation, Analysis and Applications 5 (2007), 1-27.

[4] G. Chen, R.M. Chen, Y. Liu, Existence and uniqueness of the global conservative weak solutions for the integrable Novikov equation. arXiv:1509.08569v1 [math.AP].

[5] A. Constantin, On the scattering problem for the Camassa-Holm equation, R. Soc. Lond. Proc. Ser. A Math. Phys. Eng. Sci., 457 (2001), 953-970.

[6] A. Constantin, Nonlinear water waves with applications to wave-current interactions and tsunamis. CBMS-NSF Regional Conference Series in Applied Mathematics, 81. Society for Industrial and Applied Mathematics (SIAM), Philadelphia, PA, 2011.

[7] A. Constantin and J. Escher, Wave breaking for nonlinear nonlocal shallow water equations, Acta Math. 181 (1998), 229-243.

[8] A. Constantin, R.I. Ivanov and J. Lenells, Inverse scattering transform for the DegasperisProcesi equation, Nonlinearity 23 (2010), 2559-2575.

[9] A. Constantin, H.P. McKean, A shallow water equation on the circle, Comm. Pure Appl. Math., 52 (1999), 949-982.

[10] A. Constantin, L. Molinet, Global weak solutions for a shallow water equation, Comm. Math. Phys. 211 (2000), 45-61.

[11] G.M. Coclite, K.H. Karlsen, On the well-posedness of the Degasperis-Procesi equation, J. Func. Anal., 233 (2006), 60-91.

[12] P.G. Drazin, R.S. Johnson, Solitons: an introduction. Cambridge Texts in Applied Mathematics. Cambridge University Press, Cambridge, 1989.

[13] A. Fokas, On a class of physically important integrable equations, Physica D, 87 (1995), $145-150$

[14] Y. Fu, G. Gui, Y. Liu, C. Qu, On the Cauchy problem for the integrable modified CamassaCHolm equation with cubic nonlinearity, J. Differential Equations, 255 (2013), $1905-1938$.

[15] G. Gui, Y. Liu, P. Olver, C. Qu, Wave-breaking and peakons for a modified CamassaHolm equation, Comm. Math. Phys., 319 (2013), 731-759.

[16] A.A. Himonas, D. Mantzavinos, The Cauchy problem for the Fokas-Olver-Rosenau-Qiao equation, Nonlinear Anal., 95 (2014), 499-529.

[17] C.E. Kenig, G. Ponce, L. Vega, Well-posedness and scattering results for the generalized Korteweg-de Vries equation via the contraction principle, Comm.Pure Appl. Math. , 46 (1993), 527-620.

[18] S.N. Kružhikov, First order quasi-linear equations in several independent variables, Math. USSR-Sb., 10(2) (1970), 217-243. 
[19] D. Lannes, The water waves problem. Mathematical analysis and asymptotics. Mathematical Surveys and Monographs, 188. American Mathematical Society, Providence, RI, 2013.

[20] P.J. Olver, P. Rosenau, Tri-Hamiltonian duality between solitons and solitrary-wave solutions having compact support, Phys. Rev. E, 53 (1996), 1900-1906.

[21] Z. Qiao, A new integrable equation with cuspons and W/M-shape-peaks solitons, J. Math. Phys., 47 (2006), 112701.

[22] Z. Xin and P. Zhang, On the weak solutions to a shallow water equation, Comm. Pure Appl. Math. 53 (2000), 1411-1433.

[23] Z. Xin and P. Zhang, On the uniqueness and large time behavior of the weak solutions to a shallow water equation, Comm. Partial Diff. Equat. 27 (2002), 1815-1844. 